\title{
GLOBAL WELL-POSEDNESS OF THE GROSS-PITAEVSKII AND CUBIC-QUINTIC NONLINEAR SCHRÖDINGER EQUATIONS WITH NON-VANISHING BOUNDARY CONDITIONS
}

\author{
Rowan Killip, Tadahiro Oh, Oana Pocovnicu, and Monica Vişan
}

\begin{abstract}
We consider the Gross-Pitaevskii equation on $\mathbb{R}^{4}$ and the cubic-quintic nonlinear Schrödinger equation (NLS) on $\mathbb{R}^{3}$ with non-vanishing boundary conditions at spatial infinity. By viewing these equations as perturbations to the energy-critical NLS, we prove that they are globally well-posed in their energy spaces. In particular, we prove unconditional uniqueness in the energy spaces for these equations.
\end{abstract}

\section{Introduction}

In this paper, we consider two models of nonlinear Schrödinger equation (NLS) type with non-vanishing boundary conditions at spatial infinity, namely, the GrossPitaevskii equation (1.1) in four spatial dimensions and the cubic-quintic model (1.9) in three spatial dimensions. Both models admit stable constant solutions; in this paper we study excitations around these. This constitutes the origin of the non-zero boundary conditions at spatial infinity. The common theme of the two models considered in this paper is the fact that the highest power appearing in the nonlinearity is energy-critical.

We start by discussing the Cauchy problem for the Gross-Pitaevskii equation:

$$
\left\{\begin{array}{l}
i \partial_{t} u+\Delta u=\left(|u|^{2}-1\right) u, \quad(t, x) \in \mathbb{R} \times \mathbb{R}^{n}, \\
\left.u\right|_{t=0}=u_{0},
\end{array}\right.
$$

with the non-vanishing boundary condition:

$$
\lim _{|x| \rightarrow \infty}|u(x)|=1 .
$$

The Gross-Pitaevskii equation (1.1) appears in various physical problems [6, 12, 20, 26] such as superfluidity of Helium II, Bose-Einstein condensation, and nonlinear optics ("dark" and "black" solitons, optical vortices). It constitutes the Hamiltonian evolution corresponding to the Ginzburg-Landau energy:

$$
E(u)=\frac{1}{2} \int_{\mathbb{R}^{n}}|\nabla u|^{2} d x+\frac{1}{4} \int_{\mathbb{R}^{n}}\left(|u|^{2}-1\right)^{2} d x .
$$

Received by the editors December 18, 2011.

2010 Mathematics Subject Classification. 35Q55.

Key words and phrases. NLS; Gross-Pitaevskii equation, non-vanishing boundary condition. 
The Cauchy problem for the Gross-Pitaevskii equation (1.1) (with (1.2)) was first solved on $\mathbb{R}$ by Zhidkov [32] in what are now termed Zhidkov spaces:

$$
X^{k}\left(\mathbb{R}^{n}\right):=\left\{u \in L^{\infty}\left(\mathbb{R}^{n}\right): \partial^{\alpha} u \in L^{2}\left(\mathbb{R}^{n}\right), 1 \leq|\alpha| \leq k\right\} .
$$

In spatial dimensions $n=2,3$, Béthuel and Saut [2] proved global well-posedness in $1+H^{1}\left(\mathbb{R}^{n}\right)$ and Gallo [4] proved it in Zhidkov spaces. Finally, Gérard [7,8] proved global well-posedness on $\mathbb{R}^{n}, n=1,2,3$, in the energy space, that is, the space of functions of finite Ginzburg-Landau energy. He also proved that when $n=3,4$, the energy space is given by

$$
\mathcal{E}_{\mathrm{GP}}\left(\mathbb{R}^{n}\right)=\left\{u=\alpha+v:|\alpha|=1, v \in \dot{H}^{1}\left(\mathbb{R}^{n}\right),|v|^{2}+2 \operatorname{Re}(\bar{\alpha} v) \in L^{2}\left(\mathbb{R}^{n}\right)\right\} .
$$

Moreover, if $u_{0} \in \mathcal{E}_{\mathrm{GP}}$, then the corresponding solution $u(t)$ belongs to $\mathcal{E}_{\mathrm{GP}}$ for all $t \in \mathbb{R}$. Namely, we have $u(t)=\alpha+v(t) \in \mathcal{E}_{\mathrm{GP}}$, where $v(t)$ has the same properties as $v_{0}$ and satisfies

$$
\left\{\begin{array}{l}
i \partial_{t} v+\Delta v=\left(|v|^{2}+2 \operatorname{Re}(\bar{\alpha} v)\right)(v+\alpha), \\
\left.v\right|_{t=0}=u_{0}-\alpha .
\end{array}\right.
$$

Since $|\alpha|=1$, we can write $\alpha=e^{i \theta}$. Then, by the gauge invariance of the equation under $u \mapsto e^{-i \theta} u$, we can assume that $\theta=0$ and thus $\alpha=1$. Therefore, $u=1+v$ and $v$ satisfies

$$
\left\{\begin{array}{l}
i \partial_{t} v+\Delta v=|v|^{2} v+2 \operatorname{Re}(v) v+|v|^{2}+2 \operatorname{Re}(v), \\
\left.v\right|_{t=0}=v_{0}:=u_{0}-1 .
\end{array}\right.
$$

With $u=1+v \in \mathcal{E}_{\mathrm{GP}}\left(\mathbb{R}^{4}\right)$, we have $v \in \dot{H}^{1}\left(\mathbb{R}^{4}\right)$. Then, using the Sobolev embedding $\dot{H}^{1}\left(\mathbb{R}^{4}\right) \subset L^{4}\left(\mathbb{R}^{4}\right)$ and (1.4), we obtain $\operatorname{Re}(v) \in L^{2}\left(\mathbb{R}^{4}\right)$, and therefore $v \in H_{\text {real }}^{1}\left(\mathbb{R}^{4}\right)+i \dot{H}_{\text {real }}^{1}\left(\mathbb{R}^{4}\right)$. Here, we denoted by $H_{\text {real }}^{1}\left(\mathbb{R}^{4}\right):=H^{1}\left(\mathbb{R}^{4} ; \mathbb{R}\right)$ the Sobolev space of real-valued functions. Thus, in four dimensions the energy space is given by

$$
\mathcal{E}_{\mathrm{GP}}\left(\mathbb{R}^{4}\right)=\left\{u=1+v: v \in H_{\text {real }}^{1}\left(\mathbb{R}^{4}\right)+i \dot{H}_{\text {real }}^{1}\left(\mathbb{R}^{4}\right)\right\} .
$$

In this formulation, the Hamiltonian takes the form

$$
E(u)=E(1+v)=\frac{1}{2} \int_{\mathbb{R}^{n}}|\nabla v|^{2} d x+\frac{1}{4} \int_{\mathbb{R}^{n}}\left(|v|^{2}+2 \operatorname{Re}(v)\right)^{2} d x .
$$

To simplify notation, we will simply write $E(v)$ instead of $E(1+v)$.

Our main interest is to study the global behavior of solutions to the GrossPitaevskii equation (1.1) on $\mathbb{R}^{4}$. In this paper, we prove global well-posedness in the energy space. While of less importance from a physical point of view, the fourdimensional case is very interesting from an analytical point of view. This is due to the fact that in (1.6), the nonlinearity is the sum of a cubic term and quadratic terms, and the cubic term is energy-critical $\left(\dot{H}_{x}^{1}\right.$-critical) in four space dimensions.

Previously, Gérard [7] constructed global solutions to the Gross-Pitaevskii equation on $\mathbb{R}^{4}$ for initial data in the energy space with small energy. His proof is based on 
contraction mapping arguments in Strichartz spaces; correspondingly, the uniqueness statement is for solutions in the energy space with $\nabla u \in L_{t, \text { loc }}^{2} L_{x}^{4}$. As the cubic nonlinearity is energy-critical, contraction mapping arguments cannot be used to prove global existence of solutions with large energy, even if $v_{0} \in C_{c}^{\infty}\left(\mathbb{R}^{4}\right)$.

Let us now describe briefly what is known about the long-time behavior of solutions to (1.1). Writing $\tilde{u}:=e^{-i t} u$, we obtain a solution to the defocusing cubic nonlinear Schrödinger equation

$$
i \partial_{t} \tilde{u}+\Delta \tilde{u}=|\tilde{u}|^{2} \tilde{u}
$$

with boundary condition $\lim _{|x| \rightarrow \infty}|\tilde{u}(x)|=1$. The non-vanishing boundary condition makes the long-time dynamics of the Gross-Pitaevskii equation more complex than that of the usual NLS with zero boundary condition. For instance, on $\mathbb{R}^{n}$ with $n=3,4$, solutions to the Gross-Pitaevskii equation with small energy scatter [13, 14], while at higher levels of energy, one can build traveling wave solutions $[2,3,19]$. The dynamics of the usual defocusing cubic NLS on $\mathbb{R}^{n}$ with $n=3,4$ is more straightforward: all solutions are known to scatter. In this paper, we do not address the long-time behavior of solutions to (1.1).

Our main result about the Gross-Pitaevskii equation is as follows:

Theorem 1.1. The Gross-Pitaevskii equation (1.1) is globally well-posed in the energy space $\mathcal{E}_{\mathrm{GP}}\left(\mathbb{R}^{4}\right)$. In particular, solutions are unconditionally unique in the class $C_{t}\left(\mathbb{R} ; \mathcal{E}_{\mathrm{GP}}\left(\mathbb{R}^{4}\right)\right)$.

Remark 1.2. As the proof of Theorem 1.1 yields spacetime bounds on any compact time interval, our arguments also resolve the global regularity question for (1.1), namely, that Schwartz excitations lead to global Schwartz solutions.

The proof of Theorem 1.1 is presented in Section 3. The idea of the proof is to treat (1.6) as the energy-critical cubic NLS on $\mathbb{R}^{4}$ with a subcritical perturbation, and then to use the perturbative approach developed by Tao et al. [28]. This allows us to construct global solutions to (1.1) for all initial data in the energy space. These solutions satisfy local-in-time (Strichartz) spacetime bounds. Finally, we prove unconditional uniqueness for these solutions, that is, they are unique in the larger class of solutions that are merely continuous (in time) with values in the energy space.

The second topic of this paper is the Cauchy problem for the cubic-quintic NLS with non-vanishing boundary condition:

$$
\left\{\begin{array}{l}
i \partial_{t} u+\Delta u=\alpha_{1} u-\alpha_{3}|u|^{2} u+\alpha_{5}|u|^{4} u, \quad(t, x) \in \mathbb{R} \times \mathbb{R}^{3}, \\
\left.u\right|_{t=0}=u_{0},
\end{array}\right.
$$

where $\alpha_{1}, \alpha_{3}, \alpha_{5}>0$ with $\alpha_{3}^{2}-4 \alpha_{1} \alpha_{5}>0$. This condition guarantees that the polynomial $\alpha_{1}-\alpha_{3} s+\alpha_{5} s^{2}$ has two distinct real positive roots $r_{0}^{2}>r_{1}^{2}>0$. The boundary condition is given by

$$
\lim _{|x| \rightarrow \infty}|u(x)|=r_{0} .
$$

The choice of the larger root guarantees energetic stability of the constant solution; this constitutes a local minimum of the energy functional (1.13) below. 
Equation (1.9) appears in a great variety of physical problems. It is a model in superfluidity [9, 10], descriptions of bosons [1] and of defectons [21], the theory of ferromagnetic and molecular chains [22, 23], and in nuclear hydrodynamics [15].

By rescaling (both spacetime and the values of $u$ ), it suffices to consider the case $r_{0}^{2}=1$ and $\alpha_{5}=1$. Thus, (1.9) reduces to

$$
\left\{\begin{array}{l}
i \partial_{t} u+\Delta u=\left(|u|^{2}-1\right)\left(|u|^{2}-r_{1}^{2}\right) u, \\
\left.u\right|_{t=0}=u_{0}
\end{array}\right.
$$

with the boundary condition:

$$
\lim _{|x| \rightarrow \infty}|u(x)|=1
$$

Moreover, the Hamiltonian for (1.11) is given by

$$
E(u)=\frac{1}{2} \int_{\mathbb{R}^{3}}|\nabla u|^{2} d x+\frac{\gamma}{4} \int_{\mathbb{R}^{3}}\left(|u|^{2}-1\right)^{2} d x+\frac{1}{6} \int_{\mathbb{R}^{3}}\left(|u|^{2}-1\right)^{3} d x,
$$

where $\gamma=1-r_{1}^{2}>0$. As in the case of the Gross-Pitaevskii equation, we can write our solution as $u(t)=1+v(t)$ and so obtain

$$
\left\{\begin{array}{l}
i \partial_{t} v+\Delta v=|v|^{4} v+\mathcal{R}(v), \quad(t, x) \in \mathbb{R} \times \mathbb{R}^{3}, \\
\left.v\right|_{t=0}=v_{0} .
\end{array}\right.
$$

Here, $\mathcal{R}(v)$ represents the deviation from the energy-critical NLS and is given by

$$
\begin{aligned}
\mathcal{R}(v)= & |v|^{4}+4|v|^{2} v \operatorname{Re}(v)+4|v|^{2} \operatorname{Re}(v)+\gamma|v|^{2} v+4 v \operatorname{Re}(v)^{2} \\
& +\gamma|v|^{2}+4 \operatorname{Re}(v)^{2}+2 \gamma v \operatorname{Re}(v)+2 \gamma \operatorname{Re}(v) .
\end{aligned}
$$

In this formulation, the Hamiltonian can be written as

$$
\begin{aligned}
E(u)=E(1+v)= & \frac{1}{2} \int_{\mathbb{R}^{3}}|\nabla v|^{2} d x+\frac{\gamma}{4} \int_{\mathbb{R}^{3}}\left(|v|^{2}+2 \operatorname{Re}(v)\right)^{2} d x \\
& +\frac{1}{6} \int_{\mathbb{R}^{3}}\left(|v|^{2}+2 \operatorname{Re}(v)\right)^{3} d x .
\end{aligned}
$$

As before, we will simply write $E(v)$ for $E(1+v)$.

Unlike the Hamiltonian (1.8) for the Gross-Pitaevskii equation, the Hamiltonian for the cubic-quintic NLS is not sign-definite (at least when $\gamma<2 / 3$ ). In this paper, we define the "energy space" as

$$
\mathcal{E}_{C Q}\left(\mathbb{R}^{3}\right):=1+\left(H_{\text {real }}^{1}\left(\mathbb{R}^{3}\right)+i \dot{H}_{\text {real }}^{1}\left(\mathbb{R}^{3}\right)\right) \cap L^{4}\left(\mathbb{R}^{3}\right) .
$$

Because of the lack of sign-definiteness of the energy density, this is not the unique candidate for the name "energy space". Nevertheless, $\mathcal{E}_{C Q}\left(\mathbb{R}^{3}\right)$ is the largest such candidate that we can treat by the methods of this paper. In Section 4.1, we show that $u=1+v$ belongs to the energy space $\mathcal{E}_{\mathrm{CQ}}\left(\mathbb{R}^{3}\right)$ if and only if $|E(u)|<\infty$ and $\operatorname{Re}(v) \in L^{2}\left(\mathbb{R}^{3}\right)$. This allows us to prove global well-posedness of equation (1.11) in 
$\left(H_{\text {real }}^{1}\left(\mathbb{R}^{3}\right)+i \dot{H}_{\text {real }}^{1}\left(\mathbb{R}^{3}\right)\right) \cap L^{4}\left(\mathbb{R}^{3}\right)$. As a consequence, we obtain the second main result of this paper.

Theorem 1.3. The cubic-quintic NLS (1.11) (and hence (1.9) with $\alpha_{3}^{2}-4 \alpha_{1} \alpha_{5}>$ $0)$ is globally well-posed in the energy space $\mathcal{E}_{\mathrm{CQ}}\left(\mathbb{R}^{3}\right)$. In particular, solutions are unconditionally unique in $C_{t}\left(\mathbb{R} ; \mathcal{E}_{\mathrm{CQ}}\left(\mathbb{R}^{3}\right)\right)$.

The proof of Theorem 1.3 is presented in Section 4. As for Theorem 1.1, the main ingredient in the proof of Theorem 1.3 is the perturbative approach developed in [28]. In this case, the Hamiltonian $E(v)$ in (1.16) does not control the $\dot{H}^{1}$-norm of $v$. Nonetheless, it turns out that the quantity $M(v):=E(v)+C_{0} \int|\operatorname{Re}(v)|^{2} d x$, for some $C_{0}=C_{0}(\gamma)>0$, controls the $\dot{H}^{1}$-norm of $v$. While $E(v)$ is conserved under (1.14), this new quantity $M(v)$ is no longer conserved. However, we show that $M(v(t))$ remains finite over any finite time interval for any initial data $u_{0}$ in the energy class $\mathcal{E}_{\mathrm{CQ}}\left(\mathbb{R}^{3}\right)$. This is sufficient to construct global solutions for such initial data. Finally, we establish uniqueness of solutions that are merely continuous in the energy space.

\section{Notations and Perturbation lemma}

2.1. Notations. We often use the notation $X \lesssim Y$ or $X=O(Y)$ whenever there exists some constant $C>0$ so that $X \leq C Y$. We use $X \sim Y$ if $X \lesssim Y \lesssim X$. We use $X \ll Y$ if $X \leq c Y$ for some small constant $c>0$. Note that the derivative operator $\nabla$ acts only on the spatial variables.

We say that a pair of exponents $(q, r)$ is Schrödinger-admissible if $\frac{2}{q}+\frac{n}{r}=\frac{n}{2}$ with $2 \leq q, r \leq \infty$ and $(q, r, n) \neq(2, \infty, 2)$. Given a spacetime slab $I \times \mathbb{R}^{n}$, we define the $\dot{S}^{0}\left(I \times \mathbb{R}^{n}\right)$-Strichartz norm by

$$
\|v\|_{\dot{S}^{0}(I)}=\|v\|_{\dot{S}^{0}\left(I \times \mathbb{R}^{n}\right)}:=\sup \|v\|_{L_{t}^{q} L_{x}^{r}\left(I \times \mathbb{R}^{n}\right)},
$$

where the supremum is taken over all admissible pairs $(q, r)$. We define the $\dot{S}^{1}\left(I \times \mathbb{R}^{n}\right)$ Strichartz norm by

$$
\|v\|_{\dot{S}^{1}(I)}=\|v\|_{\dot{S}^{1}\left(I \times \mathbb{R}^{n}\right)}:=\|\nabla v\|_{\dot{S}^{0}\left(I \times \mathbb{R}^{n}\right)} .
$$

We use $\dot{N}^{0}\left(I \times \mathbb{R}^{n}\right)$ to denote the dual space of $\dot{S}^{0}\left(I \times \mathbb{R}^{n}\right)$, and

$$
\dot{N}^{1}\left(I \times \mathbb{R}^{n}\right):=\left\{u: I \times \mathbb{R}^{n} \rightarrow \mathbb{C}: \nabla u \in N^{0}\left(I \times \mathbb{R}^{n}\right)\right\} .
$$

Next, we recall the Strichartz estimates; see Ginibre and Velo [11], Keel and Tao [16], Strichartz [25], and Yajima [31].

Lemma 2.1. Let $I$ be an interval in $\mathbb{R}$. Then, for $j=0,1$ we have the following homogeneous Strichartz estimate:

$$
\left\|e^{i t \Delta} u_{0}\right\|_{\dot{S}^{j}\left(I \times \mathbb{R}^{n}\right)} \lesssim\left\|u_{0}\right\|_{\dot{H}_{x}^{j}\left(\mathbb{R}^{n}\right)}
$$

and the inhomogeneous Strichartz estimate:

$$
\left\|\int_{t_{0}}^{t} e^{i\left(t-t^{\prime}\right) \Delta} F\left(t^{\prime}\right) d t^{\prime}\right\|_{\dot{S}^{j}\left(I \times \mathbb{R}^{n}\right)} \lesssim\|F\|_{\dot{N}^{j}\left(I \times \mathbb{R}^{n}\right)} .
$$


Some of the Schrödinger-admissible pairs for $n=3,4$ that we will use below are:

- $n=4:(2,4),\left(6, \frac{12}{5}\right),(\infty, 2)$,

- $n=3:(2,6),\left(\frac{8}{3}, 4\right),\left(\frac{20}{7}, \frac{30}{8}\right),\left(5, \frac{30}{11}\right),\left(10, \frac{30}{13}\right),\left(20, \frac{15}{7}\right),(\infty, 2)$.

Lastly, given an interval $I$, we use $\dot{X}^{1}(I)=\dot{X}^{1}\left(I \times \mathbb{R}^{n}\right)$ to denote the following spaces:

$$
\begin{aligned}
& \text { - } n=4:\|v\|_{\dot{X}^{1}(I)}:=\|\nabla v\|_{L_{t}^{6} L_{x}^{\frac{12}{5}}\left(I \times \mathbb{R}^{4}\right)}, \\
& \text { - } n=3:\|v\|_{\dot{X}^{1}(I)}:=\|\nabla v\|_{L_{t}^{10} L_{x}^{\frac{30}{13}}\left(I \times \mathbb{R}^{3}\right)} .
\end{aligned}
$$

2.2. Perturbation lemma. In [28], Tao et al. considered global well-posedness and scattering questions for NLS with combined power-type nonlinearities:

$$
i \partial_{t} u+\Delta u=\lambda_{1}|u|^{p_{1}} u+\lambda_{2}|u|^{p_{2}} u, \quad 0<p_{1}<p_{2} \leq \frac{4}{n-2} \quad \text { and } \quad \lambda_{1}, \lambda_{2}= \pm 1
$$

In particular, when $p_{2}=\frac{4}{n-2}$ and $\lambda_{2}=1$, they treated (2.1) as a perturbation of the defocusing energy-critical NLS:

$$
i \partial_{t} w+\Delta w=|w|^{\frac{4}{n-2}} w
$$

Global well-posedness and scattering for (2.2) was proved by Colliander et al. [5] for $n=3$ and subsequently by Ryckman and Vişan [24] and Vişan [29] for $n \geq 4$. The three and four-dimensional results (which underpin this paper) were revisited in [18] and [30] in light of recent developments. Importantly for the considerations of this paper, all these works also proved global spacetime bounds. Specifically, if $w$ is a solution to the energy-critical NLS (2.2) with initial data $w_{0} \in \dot{H}^{1}\left(\mathbb{R}^{n}\right)$, then

$$
\|w\|_{\dot{S}^{1}\left(\mathbb{R} \times \mathbb{R}^{n}\right)} \leq C\left(\left\|w_{0}\right\|_{\dot{H}^{1}}\right) .
$$

To prove global well-posedness for solutions to (2.1) (with $\lambda_{1}=\lambda_{2}=1$ and $p_{2}=$ $\left.\frac{4}{n-2}\right)$, Tao et al. combined the spacetime bounds (2.3) with a perturbation lemma [28, Lemma 3.8], which appeared in [27]. In this spirit, we regard the Gross-Pitaevskii equation (1.6) on $\mathbb{R}^{4}$ and the cubic-quintic NLS (1.14) on $\mathbb{R}^{3}$ as perturbations to (2.2) on $\mathbb{R}^{4}$ and $\mathbb{R}^{3}$, respectively. Hence, our basic strategy is also to use a perturbative approach.

The key perturbation result we will use is Lemma 2.2 below, which is [17, Theorem 3.8] and represents a strengthening of the result in [27]. The additional strength is not needed for our purposes here; however, the statement is simpler and it makes our arguments slightly simpler, too.

Lemma 2.2 (Perturbation lemma, [17]). Let $n \geq 3$ and let I be a compact time interval. Let $\tilde{w}$ be a solution on $I \times \mathbb{R}^{n}$ to the perturbed equation:

$$
i \partial_{t} \tilde{w}+\Delta \tilde{w}=|\tilde{w}|^{\frac{4}{n-2}} \tilde{w}+e
$$


for some function e. Let $w_{0} \in \dot{H}^{1}\left(\mathbb{R}^{n}\right)$ and suppose that there exist $L, E_{0}, E^{\prime}>0$ and some $t_{0} \in I$ such that

$$
\begin{aligned}
\|\tilde{w}\|_{L_{t, x}^{\frac{2(n+2)}{n-2}}\left(I \times \mathbb{R}^{n}\right)} & \leq L, \\
\|\tilde{w}\|_{L_{t}^{\infty} \dot{H}_{x}^{1}\left(I \times \mathbb{R}^{n}\right)} & \leq E_{0}, \\
\left\|\tilde{w}\left(t_{0}\right)-w_{0}\right\|_{\dot{H}^{1}\left(\mathbb{R}^{n}\right)} & \leq E^{\prime} .
\end{aligned}
$$

Furthermore, assume that we have

$$
\begin{gathered}
\left.\left\|e^{i\left(t-t_{0}\right) \Delta}\left(\tilde{w}\left(t_{0}\right)-w_{0}\right)\right\|\right|_{L_{t, x}^{\frac{2(n+2)}{n-2}}\left(I \times \mathbb{R}^{n}\right)} \leq \varepsilon, \\
\|\nabla e\|_{\dot{N}^{0}\left(I \times \mathbb{R}^{n}\right)} \leq \varepsilon,
\end{gathered}
$$

for some $0<\varepsilon \leq \varepsilon_{0}$, where $\varepsilon_{0}=\varepsilon_{0}\left(E_{0}, E^{\prime}, L\right)>0$ is a small constant. Then, the solution $w$ to $(2.2)$ with the specified initial data $w_{0}$ at time $t=t_{0}$ satisfies

$$
\begin{aligned}
\|w-\tilde{w}\|_{L_{t, x}^{\frac{2(n+2)}{n-2}}} & \leq C\left(E_{0}, E^{\prime}, L\right) \varepsilon^{c}, \\
\|w-\tilde{w}\|_{\dot{S}^{1}\left(I \times \mathbb{R}^{n}\right)} & \leq C\left(E_{0}, E^{\prime}, L\right) E^{\prime}, \\
\|w\|_{\dot{S}^{1}\left(I \times \mathbb{R}^{n}\right)} & \leq C\left(E_{0}, E^{\prime}, L\right),
\end{aligned}
$$

where $C\left(E_{0}, E^{\prime}, L\right)>0$ is a non-decreasing function of $E_{0}, E^{\prime}$, and $L$ and $c=c(n)>$ 0 .

Remark 2.3. By the Strichartz inequality together with Sobolev embedding, condition (2.8) is redundant if $E^{\prime}=O(\varepsilon)$.

We will apply this lemma with $\tilde{w}=v$, thereby showing that it differs little from a solution $w$ to (2.2). This will allow us to transfer the known spacetime bounds (2.3) for $w$ to $v$, at least locally in time. Note that in the case of the Gross-Pitaevskii equation, condition (2.6) is guaranteed by the conservation of energy (1.8). In the cubic-quintic case, the failure of the energy (1.16) to be coercive adds an additional wrinkle to the analysis.

\section{Global well-posedness of the Gross-Pitaevskii equation on $\mathbb{R}^{4}$}

In this section, we present the proof of Theorem 1.1. This breaks naturally into two parts: global existence and unconditional uniqueness.

3.1. Global existence. By time reversibility of equation (1.6), we need only consider the problem for $t \geq 0$. Let $v_{0}$ be such that $1+v_{0} \in \mathcal{E}_{\mathrm{GP}}\left(\mathbb{R}^{4}\right)$. By the usual local well-posedness arguments, it suffices to prove that there exists $T=T\left(E\left(v_{0}\right)\right)>0$ such that

$$
\|v\|_{\dot{S}^{1}\left([0, T] \times \mathbb{R}^{4}\right)} \leq C\left(E\left(v_{0}\right)\right)
$$

that is, we assume the solution $v$ exists on $[0, T]$ and prove that it satisfies (3.1). Indeed, it is not difficult to prove local existence via contraction mapping in the space 
$\dot{X}^{1}$ by using the estimates below ((3.4) in particular). Note that this argument gives local existence on any sufficiently short interval $I$ for which $\left\|e^{i t \Delta} v_{0}\right\|_{\dot{X}^{1}(I)}$ is sufficiently small. Strichartz estimates together with the monotone/dominated convergence theorems guarantee that one can always find such a short interval $I$.

Finally, combining (3.1) with conservation of the Hamiltonian $E(v)$, one can iterate the local argument, thus obtaining a global solution $v$ to (1.6) that is continuous (in time) with values in the energy space and lies in $\dot{S}^{1}(I)$ for any compact time interval $I$.

As mentioned before, the main idea is to view (1.6) as a perturbation to the energycritical cubic NLS (2.2), that is, regard $v$ as $\tilde{w}$ in Lemma 2.2 with

$$
e=2 \operatorname{Re}(v) v+|v|^{2}+2 \operatorname{Re}(v) .
$$

The argument follows closely the one in Section 4 of [28].

Let $w$ be the global solution to the energy-critical cubic NLS (2.2) with initial data $v_{0}$. Divide the interval $\mathbb{R}_{+}=[0, \infty)$ into $J=J\left(E\left(v_{0}\right), \eta\right)$ many subintervals $I_{j}=\left[t_{j}, t_{j+1}\right]$ such that

$$
\|w\|_{\dot{X}^{1}\left(I_{j}\right)}=\|\nabla w\|_{L_{t}^{6} L_{x}^{\frac{12}{5}}\left(I_{j} \times \mathbb{R}^{4}\right)} \sim \eta
$$

for some small $\eta>0$ to be chosen later.

Fix $T>0$ (to be chosen later in terms of $\left.E\left(v_{0}\right)\right)$, and write $[0, T]=\bigcup_{j=0}^{J^{\prime}}\left([0, T] \cap I_{j}\right)$ for some $J^{\prime} \leq J$, where $[0, T] \cap I_{j} \neq \emptyset$ for $0 \leq j \leq J^{\prime}$.

Since the nonlinear evolution $w$ is small on $I_{j}$, it follows that the linear evolution $e^{i\left(t-t_{j}\right) \Delta} w\left(t_{j}\right)$ is also small on $I_{j}$. Indeed, using the Duhamel formula:

$$
w(t)=e^{i\left(t-t_{j}\right) \Delta} w\left(t_{j}\right)-i \int_{t_{j}}^{t} e^{i(t-s) \Delta}|w(s)|^{2} w(s) d s, \quad \text { for } \quad t \in I_{j},
$$

together with Strichartz estimates, Sobolev embedding, and (3.2), we obtain

$$
\begin{aligned}
\left\|e^{i\left(t-t_{j}\right) \Delta} w\left(t_{j}\right)\right\|_{\dot{X}^{1}\left(I_{j}\right)} & \leq\|w\|_{\dot{X}^{1}\left(I_{j}\right)}+C\left\|w^{2} \nabla w\right\|_{L_{t}^{2} L_{x}^{\frac{4}{3}}\left(I_{j} \times \mathbb{R}^{4}\right)} \\
& \leq \eta+C\|\nabla w\|_{L_{t}^{6} \frac{12}{\frac{12}{5}}\left(I_{j} \times \mathbb{R}^{4}\right)}\|w\|_{L_{t, x}^{6}\left(I_{j} \times \mathbb{R}^{4}\right)}^{2} \\
& \leq \eta+C\|\nabla w\|_{L_{t}^{6} L_{x}^{\frac{12}{5}}\left(I_{j} \times \mathbb{R}^{4}\right)}^{3} \\
& \leq \eta+C \eta^{3},
\end{aligned}
$$

where $C$ is an absolute constant. Therefore, if $\eta$ is small enough, we obtain

$$
\left\|e^{i\left(t-t_{j}\right) \Delta} w\left(t_{j}\right)\right\|_{\dot{X}^{1}\left(I_{j}\right)} \leq 2 \eta .
$$


Now, we estimate $v$ on the first interval $I_{0}$. Arguing as before, using (3.3) and $w(0)=v(0)=v_{0}$, we obtain

$$
\begin{aligned}
\|v\|_{\dot{X}^{1}\left(I_{0}\right)} \leq & \left\|e^{i t \Delta} v_{0}\right\|_{\dot{X}^{1}\left(I_{0}\right)}+C\left\|v^{2} \nabla v\right\|_{L_{t}^{2} L_{x}^{\frac{4}{3}}\left(I_{0} \times \mathbb{R}^{4}\right)} \\
& +C\|v \nabla v\|_{L_{t}^{\frac{6}{5}} L_{x}^{\frac{12}{7}}\left(I_{0} \times \mathbb{R}^{4}\right)}+C\|\nabla v\|_{L_{t}^{1} L_{x}^{2}\left(I_{0} \times \mathbb{R}^{4}\right)} \\
\leq & \left\|e^{i t \Delta} v_{0}\right\|_{\dot{X}^{1}\left(I_{0}\right)}+C\|\nabla v\|_{L_{t}^{6} L_{x}^{\frac{12}{5}}\left(I_{0} \times \mathbb{R}^{4}\right)}^{3} \\
& +C\left|I_{0}\right|^{\frac{1}{2}}\|v\|_{L_{t, x}^{6}\left(I_{0} \times \mathbb{R}^{4}\right)}\|\nabla v\|_{L_{t}^{6} L_{x}^{\frac{12}{5}}\left(I_{0} \times \mathbb{R}^{4}\right)}+C\left|I_{0}\right|\|\nabla v\|_{L_{t}^{\infty} L_{x}^{2}\left(I_{0} \times \mathbb{R}^{4}\right)} \\
\leq & 2 \eta+C\|v\|_{\dot{X}^{1}\left(I_{0}\right)}^{3}+C T^{\frac{1}{2}}\|v\|_{\dot{X}^{1}\left(I_{0}\right)}^{2}+C T E\left(v_{0}\right)^{\frac{1}{2}} .
\end{aligned}
$$

Choosing $\eta \ll 1$ and $T \ll \min \left\{1, \eta E\left(v_{0}\right)^{-\frac{1}{2}}\right\}$, it follows from a standard continuity argument that

$$
\|v\|_{\dot{X}^{1}\left(I_{0}\right)} \leq 3 \eta
$$

Thus, by Sobolev embedding, $\|v\|_{L_{t, x}^{6}\left(I_{0} \times \mathbb{R}^{4}\right)} \leq C \eta$ and so condition (2.5) in Lemma 2.2 is satisfied with $L=C \eta$. This completes the restrictions on the small absolute constant $\eta$.

From (1.8) and the conservation of the Hamiltonian, we have

$$
\|v\|_{L_{t}^{\infty} \dot{H}_{x}^{1}\left(I_{0} \times \mathbb{R}^{4}\right)}^{2} \leq 2 E(v)=2 E\left(v_{0}\right)
$$

and thus condition (2.6) is satisfied with $E_{0}=\left[2 E\left(v_{0}\right)\right]^{1 / 2}$. With $t_{0}=0$, conditions (2.7) and (2.8) are automatically satisfied (with $E^{\prime}=0$ for (2.7)).

Let us now verify condition (2.9). Recall that $e=2 v \operatorname{Re}(v)+|v|^{2}+2 \operatorname{Re}(v)$. By the Hölder and Sobolev inequalities and (3.5), we have

$$
\begin{aligned}
& \|\nabla e\|_{\dot{N}^{0}\left(I_{0}\right)} \leq C\|v \nabla v\|_{L_{t}^{\frac{6}{5}} L_{x}^{\frac{12}{7}}\left(I_{0} \times \mathbb{R}^{4}\right)}+C\|\nabla v\|_{L_{t}^{1} L_{x}^{2}\left(I_{0} \times \mathbb{R}^{4}\right)} \\
& \leq C\left|I_{0}\right|^{\frac{1}{2}}\|\nabla v\|_{L_{t}^{6} L_{x}^{\frac{12}{5}}\left(I_{0} \times \mathbb{R}^{4}\right)}^{2}+C\left|I_{0}\right|\|\nabla v\|_{L_{t}^{\infty} L_{x}^{2}\left(I_{0} \times \mathbb{R}^{4}\right)} \\
& \leq C T^{\frac{1}{2}}\|v\|_{\dot{X}^{1}\left(I_{0}\right)}^{2}+C T E\left(v_{0}\right)^{\frac{1}{2}} \leq C T^{\frac{1}{2}} \eta^{2}+C T E\left(v_{0}\right)^{\frac{1}{2}} .
\end{aligned}
$$

Given $\varepsilon>0$, we may choose $T=T\left(E\left(v_{0}\right), \varepsilon\right)$ sufficiently small so that

$$
\|\nabla e\|_{\dot{N}^{0}\left(I_{0}\right)} \leq \varepsilon
$$

For $\varepsilon \leq \varepsilon_{0}$ with $\varepsilon_{0}=\varepsilon_{0}\left(E\left(v_{0}\right)\right)$ dictated by Lemma 2.2, condition (2.9) is thus satisfied.

Therefore, all hypotheses of Lemma 2.2 are satisfied on the interval $I_{0}$, provided $T=T\left(E\left(v_{0}\right), \varepsilon\right)$ is chosen sufficiently small. Hence, we obtain

$$
\|w-v\|_{\dot{S}^{1}\left(I_{0} \times \mathbb{R}^{4}\right)} \leq C\left(E\left(v_{0}\right)\right) \varepsilon .
$$


Next, we consider the second interval $I_{1}$. Condition (2.7) is satisfied on $I_{1}$ with $E^{\prime}=C\left(E\left(v_{0}\right)\right) \varepsilon$; indeed, (3.7) yields

$$
\left\|w\left(t_{1}\right)-v\left(t_{1}\right)\right\|_{\dot{H}^{1}\left(\mathbb{R}^{4}\right)} \leq\|w-v\|_{\dot{S}^{1}\left(I_{0} \times \mathbb{R}^{4}\right)} \leq C\left(E\left(v_{0}\right)\right) \varepsilon .
$$

In view of Remark 2.3, condition (2.8) is satisfied for $\varepsilon \leq \varepsilon_{0}\left(E\left(v_{0}\right)\right)$, where $\varepsilon_{0}\left(E\left(v_{0}\right)\right)$ is dictated by Lemma 2.2. (Note that $E^{\prime}$ grows with each successive interval $I_{j}$, but its ultimate size is $C\left(J^{\prime}, E\left(v_{0}\right)\right) \varepsilon$.)

By Strichartz with (3.8), we have

$$
\left\|e^{i\left(t-t_{1}\right) \Delta}\left(v\left(t_{1}\right)-w\left(t_{1}\right)\right)\right\|_{\dot{X}^{1}\left(I_{1}\right)} \leq C\left(E\left(v_{0}\right)\right) \varepsilon .
$$

Then, proceeding as in (3.4) and using (3.3) and (3.9),

$$
\begin{aligned}
\|v\|_{\dot{X}^{1}\left(I_{1}\right)} \leq & \left\|e^{i\left(t-t_{1}\right) \Delta} v\left(t_{1}\right)\right\|_{\dot{X}^{1}\left(I_{1}\right)}+C\|v\|_{\dot{X}^{1}\left(I_{1}\right)}^{3}+C T^{\frac{1}{2}}\|v\|_{\dot{X}^{1}\left(I_{1}\right)}^{2}+C T E\left(v_{0}\right)^{\frac{1}{2}} \\
\leq & \left\|e^{i\left(t-t_{1}\right) \Delta} w\left(t_{1}\right)\right\|_{\dot{X}^{1}\left(I_{1}\right)}+\left\|e^{i\left(t-t_{1}\right) \Delta}\left(v\left(t_{1}\right)-w\left(t_{1}\right)\right)\right\|_{\dot{X}^{1}\left(I_{1}\right)} \\
& +C\|v\|_{\dot{X}^{1}\left(I_{1}\right)}^{3}+C T^{\frac{1}{2}}\|v\|_{\dot{X}^{1}\left(I_{1}\right)}^{2}+C T E\left(v_{0}\right)^{\frac{1}{2}} \\
\leq & 2 \eta+C\left(E\left(v_{0}\right)\right) \varepsilon+C\|v\|_{\dot{X}^{1}\left(I_{1}\right)}^{3}+C T^{\frac{1}{2}}\|v\|_{\dot{X}^{1}\left(I_{1}\right)}^{2}+C T E\left(v_{0}\right)^{\frac{1}{2}} .
\end{aligned}
$$

As $\eta \ll 1$, choosing $T=T\left(E\left(v_{0}\right), \eta\right)$ and $\varepsilon=\varepsilon\left(E\left(v_{0}\right), \eta\right)$ sufficiently small, we conclude that

$$
\|v\|_{\dot{X}^{1}\left(I_{1}\right)} \leq 3 \eta
$$

by a continuity argument. Thus, by Sobolev embedding, we see that condition (2.5) in Lemma 2.2 is satisfied with $L=C \eta$ as before. Condition (2.6) is satisfied with $E_{0}=\left[2 E\left(v_{0}\right)\right]^{1 / 2}$. Lastly, as in (3.6), using (3.10), we have

$$
\|\nabla e\|_{\dot{N}^{0}\left(I_{1}\right)} \leq C T^{\frac{1}{2}}\|v\|_{\dot{X}^{1}\left(I_{1}\right)}^{2}+C T E\left(v_{0}\right)^{\frac{1}{2}} \leq C T^{\frac{1}{2}} \eta^{2}+C T E\left(v_{0}\right)^{\frac{1}{2}} \leq \varepsilon
$$

for $T=T\left(E\left(v_{0}\right), \varepsilon\right)$ sufficiently small. Therefore, condition (2.9) is satisfied, provided $\varepsilon \leq \varepsilon_{0}\left(E\left(v_{0}\right)\right)$, where $\varepsilon_{0}\left(E\left(v_{0}\right)\right)$ is dictated by Lemma 2.2 . Hence, by Lemma 2.2 applied to the interval $I_{1}$,

$$
\|v-w\|_{\dot{S}^{1}\left(I_{1}\right)} \leq C\left(E\left(v_{0}\right)\right) \varepsilon
$$

Arguing inductively, we obtain

$$
\|v\|_{\dot{X}^{1}\left(I_{j}\right)} \leq 3 \eta
$$

for all $0 \leq j \leq J^{\prime}$, provided that $\varepsilon$ and $T$ are small, depending only on $E\left(v_{0}\right)$ (and $\eta \ll 1$ and $\left.J^{\prime}\right)$. As $J^{\prime} \leq J=J\left(E\left(v_{0}\right), \eta\right)$, we obtain $T=T\left(E\left(v_{0}\right)\right)$ and

$$
\|v\|_{\dot{X}^{1}([0, T])} \leq 3 \eta J\left(E\left(v_{0}\right), \eta\right) \leq C\left(E\left(v_{0}\right)\right) .
$$


Using (3.11), we can estimate the $\dot{S}^{1}$-norm of the solution $v$ on $[0, T]$ by

$$
\begin{aligned}
\|v\|_{\dot{S}^{1}([0, T])} & \lesssim\left\|v_{0}\right\|_{\dot{H}^{1}}+\|v\|_{\dot{X}^{1}([0, T])}^{3}+T^{\frac{1}{2}}\|v\|_{\dot{X}^{1}([0, T])}^{2}+T\|\nabla v\|_{L_{t}^{\infty} L_{x}^{2}\left([0, T] \times \mathbb{R}^{4}\right)} \\
& \lesssim E\left(v_{0}\right)+C\left(E\left(v_{0}\right)\right)^{3}+T^{\frac{1}{2}} C\left(E\left(v_{0}\right)\right)^{2}+T E\left(v_{0}\right)^{\frac{1}{2}} .
\end{aligned}
$$

As $T=T\left(E\left(v_{0}\right)\right)$, we finally obtain that $\|v\|_{\dot{S}^{1}([0, T])} \leq C\left(E\left(v_{0}\right)\right)$. This allows us to iterate the local argument on time intervals of length $T=T\left(E\left(v_{0}\right)\right)>0$, thus constructing a global solution $v$ with initial data $v_{0}$ such that $1+v_{0}$ lies in the energy space $\mathcal{E}_{\mathrm{GP}}\left(\mathbb{R}^{4}\right)=H_{\text {real }}^{1}\left(\mathbb{R}^{4}\right)+i \dot{H}_{\text {real }}^{1}\left(\mathbb{R}^{4}\right)$. While the construction only yields $v(t) \in \dot{H}^{1}\left(\mathbb{R}^{4}\right)$ for $t \in \mathbb{R}$, we can easily see that $1+v(t) \in \mathcal{E}_{\mathrm{GP}}\left(\mathbb{R}^{4}\right)$ for $t \in \mathbb{R}$ from the conservation of the Hamiltonian.

3.2. Unconditional uniqueness. We turn now to showing that the global solutions constructed above are unique among those that are continuous (in time) with values in the energy space. We mimic the arguments in [5, Section 16]. To this end, let $v_{0}$ be such that $1+v_{0} \in \mathcal{E}_{\mathrm{GP}}\left(\mathbb{R}^{4}\right)$ and let $v$ be the global solution to (1.6) constructed above. In particular, $v \in \dot{S}^{1}(I)$ for any compact time interval $I$.

Let $\tilde{v}:[0, \tau] \times \mathbb{R}^{4} \rightarrow \mathbb{C}$ be a second solution to (1.6) with the same initial data such that $1+\tilde{v} \in C_{t}\left([0, \tau] ; \mathcal{E}_{\mathrm{GP}}\left(\mathbb{R}^{4}\right)\right)$ and write $\omega:=v-\tilde{v}$. As $\omega(0)=0$ and $\omega$ is continuous in time, shrinking $\tau$ if necessary, we may assume

$$
\|\operatorname{Re}(\omega)\|_{L_{t}^{\infty} H_{x}^{1}\left([0, \tau] \times \mathbb{R}^{4}\right)}+\|\operatorname{Im}(\omega)\|_{L_{t}^{\infty} \dot{H}_{x}^{1}\left([0, \tau] \times \mathbb{R}^{4}\right)} \leq \eta
$$

for a small $\eta>0$ to be chosen shortly. By Sobolev embedding, this yields

$$
\|\omega\|_{L_{t}^{\infty} L_{x}^{4}\left([0, \tau] \times \mathbb{R}^{4}\right)} \lesssim \eta
$$

in particular, $\omega \in L_{t}^{2} L_{x}^{4}\left([0, \tau] \times \mathbb{R}^{4}\right)$. Recalling that $v \in \dot{S}^{1}(I)$ for any compact time interval $I$ and further shrinking $\tau$ if necessary, we may also assume that

$$
\|v\|_{L_{t, x}^{6}\left([0, \tau] \times \mathbb{R}^{4}\right)} \leq \eta
$$

Writing

$$
\begin{gathered}
{\left[|v|^{2} v+2 \operatorname{Re}(v) v+|v|^{2}+2 \operatorname{Re}(v)\right]-\left[|\tilde{v}|^{2} \tilde{v}+2 \operatorname{Re}(\tilde{v}) \tilde{v}+|\tilde{v}|^{2}+2 \operatorname{Re}(\tilde{v})\right]} \\
\quad=O\left(|\omega|^{3}+|\omega||v|^{2}+|\omega|^{2}+|\omega||v|+|\operatorname{Re}(\omega)|\right)
\end{gathered}
$$

and using the Strichartz inequality together with (3.12), (3.13), (3.14), and Hölder, we estimate

$$
\begin{aligned}
& \|\omega\|_{L_{t}^{2} L_{x}^{4}}+\|\operatorname{Re}(\omega)\|_{L_{t}^{\infty} L_{x}^{2}} \\
& \quad \lesssim\left\|\omega^{3}\right\|_{L_{t}^{2} L_{x}^{\frac{4}{3}}}+\left\|\omega v^{2}\right\|_{L_{t}^{\frac{6}{5}} L_{x}^{\frac{12}{7}}}+\left\|\omega^{2}\right\|_{L_{t}^{1} L_{x}^{2}}+\|\omega v\|_{L_{t}^{1} L_{x}^{2}}+\|\operatorname{Re}(\omega)\|_{L_{t}^{1} L_{x}^{2}} \\
& \quad \lesssim\|\omega\|_{L_{t}^{2} L_{x}^{4}}\left[\|\omega\|_{L_{t}^{\infty} L_{x}^{4}}^{2}+\|v\|_{L_{t, x}^{6}}^{2}+\tau^{\frac{1}{2}}\|\omega\|_{L_{t}^{\infty} L_{x}^{4}}+\tau^{\frac{1}{2}}\|v\|_{L_{t}^{\infty} L_{x}^{4}}\right]+\tau\|\operatorname{Re}(\omega)\|_{L_{t}^{\infty} L_{x}^{2}} \\
& \quad \lesssim\left(\eta^{2}+\eta \tau^{\frac{1}{2}}+\tau^{\frac{1}{2}}\right)\|\omega\|_{L_{t}^{2} L_{x}^{4}}+\tau\|\operatorname{Re}(\omega)\|_{L_{t}^{\infty} L_{x}^{2}} .
\end{aligned}
$$


All spacetime norms in the display above are taken on $[0, \tau] \times \mathbb{R}^{4}$. Taking $\eta$ sufficiently small and shrinking $\tau$ further if necessary, we obtain

$$
\|\omega\|_{L_{t}^{2} L_{x}^{4}\left([0, \tau] \times \mathbb{R}^{4}\right)}+\|\operatorname{Re}(\omega)\|_{L_{t}^{\infty} L_{x}^{2}\left([0, \tau] \times \mathbb{R}^{4}\right)}=0,
$$

which proves $v=\tilde{v}$ almost everywhere on $[0, \tau] \times \mathbb{R}^{4}$.

By time translation invariance, this argument can be applied to any sufficiently short time interval, which yields global unconditional uniqueness. This completes the proof of Theorem 1.1.

\section{Global well-posedness of the cubic-quintic NLS on $\mathbb{R}^{3}$}

4.1. The energy space and energy estimates. Let $E(v)$ be the Hamiltonian for (1.14) given in (1.16). It is not sign-definite in general and it does not seem to control the $\dot{H}_{x}^{1}$-norm of $v$. However, once we add a multiple of the $L_{x}^{2}$-norm of $\operatorname{Re}(v)$, the following lemma shows that it indeed controls the $\dot{H}_{x}^{1}$-norm of $v$.

Lemma 4.1. Let $E(v)$ be the Hamiltonian as in (1.16). Then, there exists $C_{0}=$ $C_{0}(\gamma)>0$ such that

$$
\int_{\mathbb{R}^{3}}|\nabla v|^{2} d x+\int_{\mathbb{R}^{3}}|v|^{6} d x+\gamma \int_{\mathbb{R}^{3}}|v|^{4} d x \lesssim E(v)+C_{0} \int_{\mathbb{R}^{3}}|\operatorname{Re}(v)|^{2} d x .
$$

Proof. By writing $|v|^{2}=\left(|v|^{2}+2 \operatorname{Re}(v)\right)-2 \operatorname{Re}(v)$, we obtain

$$
\gamma|v|^{4} \leq 2 \gamma\left(|v|^{2}+2 \operatorname{Re}(v)\right)^{2}+8 \gamma|\operatorname{Re}(v)|^{2} \leq 8\left[\frac{\gamma}{4}\left(|v|^{2}+2 \operatorname{Re}(v)\right)^{2}+\gamma|\operatorname{Re}(v)|^{2}\right]
$$

For $a, b \in \mathbb{R}$ with $a+b \geq 0$, we have the inequality $(a+b)^{3} \leq 4 a^{3}+4 b^{3}$. From this, we obtain

$$
|v|^{6}=\left[|v|^{2}+2 \operatorname{Re}(v)+(-2 \operatorname{Re}(v))\right]^{3} \leq 4\left(|v|^{2}+2 \operatorname{Re}(v)\right)^{3}+32|\operatorname{Re}(v)|^{3} .
$$

By Young's inequality,

$$
|\operatorname{Re}(v)|^{3} \leq \frac{3}{4 \delta^{\frac{4}{3}}}|\operatorname{Re}(v)|^{2}+\frac{\delta^{4}}{4}|v|^{6},
$$

for any $\delta>0$. Combining these with $\delta=1 / 2$, we obtain

$$
|v|^{6} \leq 8\left(|v|^{2}+2 \operatorname{Re}(v)\right)^{3}+144|\operatorname{Re}(v)|^{2} \leq 48\left[\frac{1}{6}\left(|v|^{2}+2 \operatorname{Re}(v)\right)^{3}+3|\operatorname{Re}(v)|^{2}\right] .
$$

Collecting (4.2) and (4.3) and using the formula for the Hamiltonian (1.16), we derive the claim.

In view of this lemma, we can characterize the energy space $\mathcal{E}_{C Q}\left(\mathbb{R}^{3}\right)$ introduced in (1.17) in terms of $E(v)$ and $\|\operatorname{Re}(v)\|_{L_{x}^{2}}$ only:

Lemma 4.2. Let $E(v)$ be as in (1.16). Then $1+v$ belongs to the energy space $\mathcal{E}_{C Q}\left(\mathbb{R}^{3}\right)$ if and only if $|E(v)|<\infty$ and $\operatorname{Re}(v) \in L^{2}\left(\mathbb{R}^{3}\right)$. 
Proof. Using Sobolev embedding, it is easy to see that if $1+v$ belongs to $\mathcal{E}_{C Q}\left(\mathbb{R}^{3}\right)$, then we have $|E(v)|<\infty$ and $\operatorname{Re}(v) \in L^{2}\left(\mathbb{R}^{3}\right)$. The converse follows from Lemma 4.1.

While the Hamiltonian $E(v)$ is conserved under the flow of (1.14), the $L_{x}^{2}$-norm of $\operatorname{Re}(v)$ is not. The following lemma controls the growth of this quantity for solutions in the energy space $\mathcal{E}_{C Q}\left(\mathbb{R}^{3}\right)$.

Lemma 4.3. Let $v$ be a solution to the cubic-quintic $N L S$ (1.14) on a time interval $[0, \tau]$ with initial data $v_{0}$ such that $1+v_{0} \in \mathcal{E}_{C Q}\left(\mathbb{R}^{3}\right)$. Then $M(v):=E(v)+$ $C_{0} \int_{\mathbb{R}^{3}}|\operatorname{Re}(v)|^{2} d x$ (with $C_{0}$ as in Lemma 4.1) satisfies the following growth estimate:

$$
M(v(t)) \leq M\left(v_{0}\right) e^{C_{1} t}
$$

for all $t \in[0, \tau]$ and some $C_{1}>0$.

Moreover, there exists $C\left(E\left(v_{0}\right),\left\|\operatorname{Re}\left(v_{0}\right)\right\|_{L^{2}\left(\mathbb{R}^{3}\right)}, \tau\right)>0$ such that

$$
\sup _{t \in[0, \tau]}\|v(t)\|_{\mathcal{E}_{C Q}\left(\mathbb{R}^{3}\right)} \leq C\left(E\left(v_{0}\right),\left\|\operatorname{Re}\left(v_{0}\right)\right\|_{L^{2}\left(\mathbb{R}^{3}\right)}, \tau\right),
$$

where

$$
\|f\|_{\mathcal{E}_{C Q}\left(\mathbb{R}^{3}\right)}:=\|\operatorname{Re}(f)\|_{H^{1}\left(\mathbb{R}^{3}\right)}+\|\operatorname{Im}(f)\|_{\dot{H}^{1}\left(\mathbb{R}^{3}\right)}+\|f\|_{L^{4}\left(\mathbb{R}^{3}\right)} .
$$

In particular, the $\dot{H}^{1}$-norm of the solution does not blow up in finite time.

Proof. We prove the lemma for smooth $v$. From (1.14), we have

$$
\begin{aligned}
\partial_{t} \int_{\mathbb{R}^{3}}|\operatorname{Re}(v)|^{2} d x= & 2 \int_{\mathbb{R}^{3}} \operatorname{Re}(v) \partial_{t} \operatorname{Re}(v) d x=-2 \int_{\mathbb{R}^{3}} \operatorname{Re}(v) \operatorname{Im}(\Delta v) d x \\
& +2 \int_{\mathbb{R}^{3}} \operatorname{Re}(v) \operatorname{Im}\left(|v|^{4} v+\mathcal{R}(v)\right) d x=: \mathrm{I}+\mathrm{II} .
\end{aligned}
$$

Integrating by parts, we have

$$
|\mathrm{I}|=\left|2 \int_{\mathbb{R}^{3}} \operatorname{Re}(\nabla v) \cdot \operatorname{Im}(\nabla v) d x\right| \lesssim \int_{\mathbb{R}^{3}}|\nabla v|^{2} d x,
$$

while from (1.15), we have

$$
\begin{aligned}
\mathbb{I I}= & 2 \int_{\mathbb{R}^{3}} \operatorname{Re}(v)|v|^{4} \operatorname{Im}(v)+4 \operatorname{Re}(v)^{2}|v|^{2} \operatorname{Im}(v) \\
& \quad+\gamma \operatorname{Re}(v)|v|^{2} \operatorname{Im}(v)+4 \operatorname{Re}(v)^{3} \operatorname{Im}(v)+2 \gamma \operatorname{Re}(v)^{2} \operatorname{Im}(v) d x \\
= & : \Pi_{1}+\Pi_{2}+\Pi_{3}+\Pi_{4}+\Pi_{5} .
\end{aligned}
$$

Trivially, $\left|\Pi_{1}\right| \lesssim \int_{\mathbb{R}^{3}}|v|^{6} d x$. By interpolation and Young's inequality,

$$
\left|\Pi_{2}\right| \lesssim \int_{\mathbb{R}^{3}}|\operatorname{Re}(v)|^{4} d x+\int_{\mathbb{R}^{3}}|v|^{6} d x \lesssim \int_{\mathbb{R}^{3}}|\operatorname{Re}(v)|^{2} d x+\int_{\mathbb{R}^{3}}|v|^{6} d x .
$$

Next, by Cauchy's inequality $\left(a b \leq \frac{a^{2}}{2}+\frac{b^{2}}{2}\right.$ for $\left.a, b \in \mathbb{R}\right)$,

$$
\left|\Pi_{3}\right|+\left|\Pi_{4}\right| \lesssim \int_{\mathbb{R}^{3}}|\operatorname{Re}(v)|^{2} d x+\int_{\mathbb{R}^{3}}|v|^{6} d x
$$


and

$$
\left|\Pi_{5}\right| \lesssim \int_{\mathbb{R}^{3}}|\operatorname{Re}(v)|^{2} d x+\int_{\mathbb{R}^{3}}|v|^{4} d x
$$

Collecting (4.6) through (4.10) and using conservation of energy and Lemma 4.1, we conclude that

$$
\partial_{t} M(v(t))=C_{0} \partial_{t} \int_{\mathbb{R}^{3}}|\operatorname{Re}(v)|^{2} d x \leq C_{1}\left[E(v)+C_{0} \int_{\mathbb{R}^{3}}|\operatorname{Re}(v)|^{2} d x\right]=C_{1} M(v(t)) .
$$

This yields (4.4). Finally, (4.5) follows from (4.4) and Lemma 4.1.

We now turn to the proof of Theorem 1.3, which we again divide into two pieces: global existence and unconditional uniqueness.

4.2. Global existence. As in Section 3, we view (1.14) as a perturbed energycritical quintic NLS on $\mathbb{R}^{3}$ with the perturbation $e=\mathcal{R}(v)$ defined in (1.15) and apply Lemma 2.2. The argument is basically the same as that in Section 3 with one difference: as the conserved Hamiltonian $E(v)$ no longer controls the $\dot{H}_{x}^{1}$-norm of the solution, we have to rely instead on Lemma 4.3. Note that the bound is not uniform in time; however, by Lemma 4.3,

$$
\|v(t)\|_{\dot{H}^{1}\left(\mathbb{R}^{3}\right)} \leq C\left(E\left(v_{0}\right),\left\|\operatorname{Re}\left(v_{0}\right)\right\|_{L^{2}\left(\mathbb{R}^{3}\right)}, \tau\right)=: N\left(v_{0}, \tau\right),
$$

for any $0 \leq t \leq \tau$ for which the solution $v$ exists.

Let $v_{0}$ be such that $1+v_{0} \in \mathcal{E}_{\mathrm{CQ}}\left(\mathbb{R}^{3}\right)$ and fix $\tau>0$. Our goal is to construct a solution $v$ to $(1.14)$ on $[0, \tau]$. It suffices to prove that there exists $T=T\left(N\left(v_{0}, \tau\right)\right)>0$ such that

$$
\|v\|_{\dot{S}^{1}\left(\left[T_{0}, T_{0}+T\right] \times \mathbb{R}^{3}\right)} \leq C\left(N\left(v_{0}, \tau\right)\right)
$$

as long as $\left[T_{0}, T_{0}+T\right] \subset[0, \tau]$. This allows us to iterate the local argument and extend the solution $v$ to $[0, \tau]$. Since the choice of $\tau>0$ is arbitrary, this yields a global solution $v$ to (1.11), which is unique in $\dot{S}_{\text {loc }}^{1}$.

Fix $T>0$ to be chosen later (depending on $N\left(v_{0}, \tau\right)$ ) and suppose $T_{0} \geq 0$ is such that $\left[T_{0}, T_{0}+T\right] \subset[0, \tau]$. Let $w$ be the global solution to the energy-critical quintic NLS (2.2) with initial data $v\left(T_{0}\right)$. As before, we divide $\left[T_{0}, \infty\right)$ into $J=$ $J\left(E\left(v\left(T_{0}\right)\right), \eta\right)=J\left(N\left(v_{0}, \tau\right), \eta\right)$ many subintervals $I_{j}=\left[t_{j}, t_{j+1}\right]$ such that

$$
\|w\|_{\dot{X}^{1}\left(I_{j}\right)}=\|\nabla w\|_{L_{t}^{10} L_{x}^{\frac{30}{13}}\left(I_{j} \times \mathbb{R}^{3}\right)} \sim \eta
$$

for some small $\eta>0$ to be chosen later. We write $\left[T_{0}, T_{0}+T\right]=\bigcup_{j=0}^{J^{\prime}}\left(\left[T_{0}, T_{0}+T\right] \cap I_{j}\right)$ for some $J^{\prime} \leq J$, where $\left[T_{0}, T_{0}+T\right] \cap I_{j} \neq \emptyset$ for $0 \leq j \leq J^{\prime}$. 
By the Strichartz estimate, Sobolev embedding, and (4.13), we have

$$
\begin{aligned}
\left\|e^{i\left(t-t_{j}\right) \Delta} w\left(t_{j}\right)\right\|_{\dot{X}^{1}\left(I_{j}\right)} & \leq\|w\|_{\dot{X}^{1}\left(I_{j}\right)}+C\left\|w^{4} \nabla w\right\|_{L_{t}^{2} L_{x}^{\frac{6}{5}}\left(I_{j} \times \mathbb{R}^{3}\right)} \\
& \leq \eta+C\|\nabla w\|_{L_{t}^{10} L_{x}^{\frac{30}{13}}\left(I_{j} \times \mathbb{R}^{3}\right)}\|w\|_{L_{t, x}^{10}\left(I_{j} \times \mathbb{R}^{3}\right)}^{4} \\
& \leq \eta+C\|\nabla w\|_{L_{t}^{10} L_{x}^{\frac{30}{13}}\left(I_{j} \times \mathbb{R}^{3}\right)} \\
& \leq \eta+C \eta^{5},
\end{aligned}
$$

where $C$ is an absolute constant. Therefore, if $\eta$ is small enough, we obtain

$$
\left\|e^{i\left(t-t_{j}\right) \Delta} w\left(t_{j}\right)\right\|_{\dot{X}^{1}\left(I_{j}\right)} \leq 2 \eta .
$$

Arguing similarly and using (4.11) and (4.14), we obtain

$$
\begin{aligned}
\|v\|_{\dot{X}^{1}\left(I_{0}\right)} \leq & \left\|e^{i t \Delta} v\left(T_{0}\right)\right\|_{\dot{X}^{1}\left(I_{0}\right)}+C\left\|v^{4} \nabla v\right\|_{L_{t}^{2} L_{x}^{\frac{6}{5}}\left(I_{0} \times \mathbb{R}^{3}\right)}+C\left\|v^{3} \nabla v\right\|_{L_{t}^{\frac{20}{13}} L_{x}^{\frac{30}{22}}\left(I_{0} \times \mathbb{R}^{3}\right)} \\
& +C\left\|v^{2} \nabla v\right\|_{L_{t}^{\frac{5}{4}} L_{x}^{\frac{30}{19}}\left(I_{0} \times \mathbb{R}^{3}\right)}+C\|v \nabla v\|_{L_{t}^{\frac{20}{19}} L_{x}^{\frac{30}{16}}\left(I_{0} \times \mathbb{R}^{3}\right)}+C\|\nabla v\|_{L_{t}^{1} L_{x}^{2}\left(I_{0} \times \mathbb{R}^{3}\right)} \\
\leq & \left\|e^{i t \Delta} v\left(T_{0}\right)\right\|_{\dot{X}^{1}\left(I_{0}\right)}+C \sum_{\ell=2}^{5}\left|I_{0}\right|^{\frac{5-\ell}{4}}\|v\|_{L_{t, x}^{10}\left(I_{0} \times \mathbb{R}^{3}\right)}^{\ell-\nabla v\left\|_{L_{t}^{10} L_{x}^{\frac{30}{13}}\left(I_{0} \times \mathbb{R}^{3}\right)}\right\|} \\
& +C\left|I_{0}\right|\|\nabla v\|_{L_{t}^{\infty} L_{x}^{2}\left(I_{0} \times \mathbb{R}^{3}\right)} \\
\leq & 2 \eta+C \sum_{\ell=2}^{5} T^{\frac{5-\ell}{4}}\|v\|_{\dot{X}^{1}\left(I_{0}\right)}^{\ell}+C T N\left(v_{0}, \tau\right) .
\end{aligned}
$$

Choosing $\eta \ll 1$ and $T \ll \min \left\{1, \frac{\eta}{N\left(v_{0}, \tau\right)}\right\}$, it follows from a standard continuity argument that

$$
\|v\|_{\dot{X}^{1}\left(I_{0}\right)} \leq 3 \eta
$$

Then, by Sobolev embedding, $\|v\|_{L_{t}^{10}\left(I_{0} \times \mathbb{R}^{3}\right)} \leq C \eta$ and so condition (2.5) in Lemma 2.2 is satisfied with $L=C \eta$. As in Section $3, \eta$ is a small absolute constant.

By (4.11), condition (2.6) is satisfied with $E_{0}=N\left(v_{0}, \tau\right)$. With $t_{0}=T_{0}$, conditions (2.7) and (2.8) are automatically satisfied (with $E^{\prime}=0$ for (2.7)).

Let us now verify condition (2.9). Recall that the perturbation $e$ in Lemma 2.2 is $e=\mathcal{R}(v)$ defined in (1.15). Proceeding as in (4.15) and using (4.16), we have

$$
\begin{aligned}
\|\nabla e\|_{\dot{N}^{0}\left(I_{0}\right)} & \leq C \sum_{\ell=2}^{4} T^{\frac{5-\ell}{4}}\|v\|_{\dot{X}^{1}\left(I_{0}\right)}^{\ell}+C T N\left(v_{0}, \tau\right) \\
& \leq C \sum_{\ell=2}^{4} T^{\frac{5-\ell}{4}} \eta^{\ell}+C T N\left(v_{0}, \tau\right) .
\end{aligned}
$$

For any $\varepsilon>0$, we may choose $T=T\left(N\left(v_{0}, \tau\right), \varepsilon\right)$ sufficiently small so that

$$
\|\nabla e\|_{\dot{N}^{0}\left(I_{0}\right)} \leq \varepsilon \text {. }
$$


Hence, condition (2.9) holds provided $\varepsilon \leq \varepsilon_{0}\left(N\left(v_{0}, \tau\right)\right)$ where $\varepsilon_{0}\left(N\left(v_{0}, \tau\right)\right)$ is dictated by Lemma 2.2 .

Therefore, all hypotheses of Lemma 2.2 are satisfied on the interval $I_{0}$, provided that $T=T\left(N\left(v_{0}, \tau\right), \varepsilon\right)$ is sufficiently small. Thus, we obtain

$$
\|w-v\|_{\dot{S}^{1}\left(I_{0} \times \mathbb{R}^{3}\right)} \leq C\left(N\left(v_{0}, \tau\right)\right) \varepsilon .
$$

The rest of the argument follows exactly as in Section 3; we omit the details. At the end of the day, we obtain

$$
\|v\|_{\dot{S}^{1}\left(\left[T_{0}, T_{0}+T\right] \times \mathbb{R}^{3}\right)} \leq C\left(N\left(v_{0}, \tau\right)\right)=C\left(E\left(v_{0}\right),\left\|\operatorname{Re}\left(v_{0}\right)\right\|_{L^{2}\left(\mathbb{R}^{3}\right)}, \tau\right),
$$

which settles (4.12) and thus the global existence question.

4.3. Unconditional uniqueness. We turn now to showing that the global solutions constructed above are unique among those that are continuous (in time) with values in the energy space. The proof is similar to that for the Gross-Pitaevski equation given in Section 3. Let $v_{0}$ be such that $1+v_{0} \in \mathcal{E}_{\mathrm{CQ}}\left(\mathbb{R}^{3}\right)$ and let $v$ be the global solution to (1.14) constructed above. In particular, $v \in \dot{S}^{1}(I)$ for any compact time interval $I$.

Let $\tilde{v}:[0, \tau] \times \mathbb{R}^{3} \rightarrow \mathbb{C}$ be a second solution to (1.14) with the same initial condition such that $1+\tilde{v} \in C_{t}\left([0, \tau] ; \mathcal{E}_{\mathrm{CQ}}\left(\mathbb{R}^{3}\right)\right)$ and write $\omega:=v-\tilde{v}$. Arguing as in the previous section and shrinking $\tau$ if necessary, we may assume

$$
\begin{aligned}
& \|v\|_{L_{t, x}^{10}\left([0, \tau] \times \mathbb{R}^{3}\right)}+\|\omega\|_{L_{t}^{\infty} L_{x}^{6}\left([0, \tau] \times \mathbb{R}^{3}\right)}+\|\omega\|_{L_{t}^{\infty} L_{x}^{4}\left([0, \tau] \times \mathbb{R}^{3}\right)} \\
& \quad+\|\operatorname{Re}(\omega)\|_{L_{t}^{\infty} H_{x}^{1}\left([0, \tau] \times \mathbb{R}^{3}\right)}+\|\operatorname{Im}(\omega)\|_{L_{t}^{\infty} \dot{H}_{x}^{1}\left([0, \tau] \times \mathbb{R}^{3}\right)} \leq \eta
\end{aligned}
$$

for a small $\eta>0$ to be chosen shortly. In particular, we have $\omega \in L_{t}^{2} L_{x}^{6}\left([0, \tau] \times \mathbb{R}^{3}\right) \cap$ $L_{t}^{\frac{8}{3}} L_{x}^{4}\left([0, \tau] \times \mathbb{R}^{3}\right)$.

Writing

$$
\left[|v|^{4} v+\mathcal{R}(v)\right]-\left[|\tilde{v}|^{4} \tilde{v}+\mathcal{R}(\tilde{v})\right]=O\left(|\omega|^{5}+|\omega||v|^{4}+|\omega|^{2}+|\omega||v|+|\operatorname{Re}(\omega)|\right)
$$

and using the Strichartz inequality together with (4.19) and Hölder, we estimate

$$
\begin{aligned}
& \|\omega\|_{L_{t}^{2} L_{x}^{6}}+\|\omega\|_{L_{t}^{\frac{8}{3}} L_{x}^{4}}+\|\operatorname{Re}(\omega)\|_{L_{t}^{\infty} L_{x}^{2}} \\
& \lesssim\left\|\omega^{5}\right\|_{L_{t}^{2} L_{x}^{\frac{6}{5}}}+\left\|\omega v^{4}\right\|_{L_{t}^{\frac{10}{9}} L_{x}^{\frac{30}{17}}}+\left\|\omega^{2}\right\|_{L_{t}^{1} L_{x}^{2}}+\|\omega v\|_{L_{t}^{1} L_{x}^{2}}+\|\operatorname{Re}(\omega)\|_{L_{t}^{1} L_{x}^{2}} \\
& \lesssim\|\omega\|_{L_{t}^{2} L_{x}^{6}}\left[\|\omega\|_{L_{t}^{\infty} L_{x}^{6}}^{4}+\|v\|_{L_{t, x}^{10}}^{4}\right] \\
& \quad+\tau^{\frac{5}{8}}\|\omega\|_{L_{t}^{\frac{8}{3}} L_{x}^{4}}\left[\|\omega\|_{L_{t}^{\infty} L_{x}^{4}}+\|v\|_{L_{t}^{\infty} L_{x}^{4}}\right]+\tau\|\operatorname{Re}(\omega)\|_{L_{t}^{\infty} L_{x}^{2}} \\
& \lesssim \eta^{4}\|\omega\|_{L_{t}^{2} L_{x}^{6}}+\tau^{\frac{5}{8}}\|\omega\|_{L_{t}^{\frac{8}{3}} L_{x}^{4}}+\tau\|\operatorname{Re}(\omega)\|_{L_{t}^{\infty} L_{x}^{2} .}
\end{aligned}
$$

All spacetime norms in the display above are taken on $[0, \tau] \times \mathbb{R}^{3}$. Taking $\eta$ sufficiently small and shrinking $\tau$ further if necessary, we obtain

$$
\|\omega\|_{L_{t}^{2} L_{x}^{6}\left([0, \tau] \times \mathbb{R}^{3}\right)}+\|\omega\|_{L_{t}^{\frac{8}{3}} L_{x}^{4}\left([0, \tau] \times \mathbb{R}^{3}\right)}+\|\operatorname{Re}(\omega)\|_{L_{t}^{\infty} L_{x}^{2}\left([0, \tau] \times \mathbb{R}^{3}\right)}=0,
$$

which proves $v=\tilde{v}$ almost everywhere on $[0, \tau] \times \mathbb{R}^{3}$.

This completes the proof of unconditional uniqueness and thus the proof of Theorem 1.3. 


\section{Acknowledgments}

We are grateful to Professor P. Gérard for bringing this problem to our attention. R. K. was partially supported by NSF grant no. DMS-1001531. T. O. acknowledges support from an AMS-Simons Travel Grant. M. V. was partially supported by the Sloan Foundation and NSF grant no. DMS-0901166.

\section{References}

[1] I.V. Barashenkov, A.D. Gocheva, V.G. Makhankov and I.V. Puzynin, Stability of the soliton-like "bubbles", Physica D 34(1-2) (1989), 240-254.

[2] F. Béthuel and J.-C. Saut, Travelling waves for the Gross-Pitaevskii equation I, Ann. Inst. H. Poincaré Phys. Théor. 70(2) (1999), 147-238.

[3] F. Béthuel, P. Gravejat and J.-C. Saut, Travelling waves for the Gross-Pitaevskii equation II, Commun. Math. Phys. 285 (2009), 567-651.

[4] C. Gallo, Schrödinger group on Zhidkov spaces, Adv. Differential Equations 9(5-6) (2004), 509538.

[5] J. Colliander, M. Keel, G. Staffilani, H. Takaoka and T. Tao, Global well-posedness and scattering for the energy-critical nonlinear Schrödinger equation in $\mathbb{R}^{3}$, Ann. Math. (2) 167(3) (2008), 767-865.

[6] T. Frisch, Y. Pomeau and S. Rica, Transition to dissipation in a model of superflow, Phys. Rev. Lett. 69 (1992), 1644-1647.

[7] P. Gérard, The Cauchy problem for the Gross-Pitaevskii equation, Ann. Inst. H. Poincaré Anal. Non Linéaire 23(5) (2006), 765-779.

[8] P. Gérard, The Gross-Pitaevskii equation in the energy space. Stationary and time dependent Gross-Pitaevskii equations, Contemp. Math. 473, Amer. Math. Soc., Providence, RI, (2008), 129-148.

[9] V.L. Ginzburg and L.P. Pitaevskii, On the theory of superfluidity, Sov. Phys. JETP 34 (1958), no. 7, 858-861; translation from Zh. Eksp. Teor. Fiz., 32 (1958), 1240-1245.

[10] V.L. Ginzburg and A.A. Sobyanin, Superfluidity of Helium II near the $\lambda$-point, Usp. Fiz. Nauk 120 (1976), 153.

[11] J. Ginibre and G. Velo, Smoothing properties and retarded estimates for some dispersive evolution equations, Comm. Math. Phys. 144(1) (1992), 163-188.

[12] E.P. Gross, Structure of a quantized vortex in boson systems, Il Nuovo Cimento 20 (1961), 454-457.

[13] S. Gustafson, K. Nakanishi and T.P. Tsai, Scattering theory for the Gross-Pitaevskii equation in three dimensions, Commun. Contemp. Math. 11(4) (2009), 657-707.

[14] S. Gustafson, K. Nakanishi and T.P. Tsai, Scattering for the Gross-Pitaevskii equation, Math. Res. Letters 13(2) (2006), 273-285.

[15] V.G. Kartavenko, Soliton-like solutions in nuclear hydrodynamics, Sov. J. Nucl. Phys. 40 (1984), 240-246; translation from Yad. Fiz. 40 (1984), 377-388.

[16] M. Keel and T. Tao, Endpoint Strichartz estimates, Amer. J. Math. 120(5) (1998), 955-980.

[17] R. Killip and M. Vişan, Nonlinear Schrödinger equations at critical regularity, Lecture notes prepared for the Clay Mathematics Institute Summer School, Zürich, Switzerland, 2008.

[18] R. Killip and M. Vişan, Global well-posedness and scattering for the defocusing quintic NLS in three dimensions, Analysis and PDE 5 (2012), 855-885.

[19] M. Mariş, Travelling waves for nonlinear Schrödinger equations with nonzero conditions at infinity, Preprint arXiv:0903.0354.

[20] L.P. Pitaevskii, Vortex lines in an imperfect Bose gas, Sov. Phys. JETP 13 (1961), 451-454.

[21] D.I. Pushkarov and Zl. Kojnov, Contribution to the theory of defectons in quantum crystals, Sov. Phys. JETP 47 (1978), 962-966; (translation from Zh. Eksp. Teor. Fiz. 74 (1978), 1845-1852).

[22] Kh.I. Pushkarov and M.T. Primatarova, Solitary clusters of spin deviations and lattice deformation in an anharmonic ferromagnetic chain, Phys. Stat. Sol. (b) 123 (1984), 573-584.

[23] Kh.I. Pushkarov and M.T. Primatarova, Solitary excitations in molecular chains in the presence of vibronic states, Phys. Stat. Sol. (b) 133 (1986), 253-265. 
[24] E. Ryckman and M. Vişan, Global well-posedness and scattering for the defocusing energycritical nonlinear Schrödinger equation in $\mathbb{R}^{1+4}$, Amer. J. Math. 129(1) (2007), 1-60.

[25] R.S. Strichartz, Restrictions of Fourier transforms to quadratic surfaces and decay of solutions of wave equations, Duke Math. J. 44(3) (1977), 705-714.

[26] C. Sulem and P.L. Sulem, The nonlinear Schrödinger equation. Self-focusing and wave collapse, Applied Mathematical Sciences, Vol. 139, Springer-Verlag, New York, 1999.

[27] T. Tao and M. Vişan, Stability of energy-critical nonlinear Schrödinger equations in high dimensions, Electron. J. Diff. Eqns. 2005 (2005), 1-28.

[28] T. Tao, M. Vişan and X. Zhang, The nonlinear Schrödinger equation with combined power-type nonlinearities, Comm. Partial Differ. Equ. 32(7-9) (2007), 1281-1343.

[29] M. Vişan, The defocusing energy-critical nonlinear Schrödinger equation in higher dimensions, Duke Math. J. 138 (2007), 281-374.

[30] M. Vişan, Global well-posedness and scattering for the defocusing cubic nonlinear Schrödinger equation in four dimensions, Int. Math. Res. Notices (2011) doi: 10.1093/imrn/rnr051.

[31] K. Yajima, Existence of solutions for Schrödinger evolution equations, Comm. Math. Phys. 110(3) (1987), 415-426.

[32] P.E. Zhidkov, The Cauchy problem for the nonlinear Schrödinger equation, Joint Inst. Nucl. Res., Dubna (1987), 15 pp.

Department of Mathematics, University of California, Los Angeles, CA 90095, USA

E-mail address: killip@math.ucla.edu

Department of Mathematics, Princeton University, Fine Hall Washington Rd., Princeton, NJ 08544-1000, USA

E-mail address: hirooh@math.princeton.edu

Department of Mathematics, Princeton University, Fine Hall, Washington Road, PRINCETON NJ 08544-1000, USA

E-mail address: opocovnicu@math.princeton.edu

Department of Mathematics, University of California, Los Angeles, CA 90095, USA

E-mail address: visan@math.ucla.edu 